\title{
Decentralized, Modular Real-Time Control for Machining Applications*
}

\author{
John Yook, Dawn Tilbury, \\ Kalyani Chervela*, Nandit Soparkar* \\ The University of Michigan \\ Mechanical Engineering and Applied Mechanics \\ *Electrical Engineering and Computer Science \\ Ann Arbor, MI 48109 \\ \{jyook,tilbury,kvc,soparkar\}@umich.edu
}

September 25, 1997

\begin{abstract}
In order to realize the vision of reconfigurable manufacturing systems, the machine tool hardware, as well as the software which controls it, must be constructed in a modular fashion. We envision that each hardware module, be it a single spindle, linear or rotary axis, or a multi-axis grouping, will have its own sensors as well as control hardware and software modules. When a set of modules are grouped together to form a machine, the control task may demand not only certain requirements for individual axes, but also have constraints on the coordination of interacting axes (as in a contouring application). Thus, the axis-level control modules, running on distributed processors with communication over a network, must be coordinated in an appropriate way to ensure that the desired task is completed with the highest possible speed and accuracy. This coordination gives rise to stringent constraints on both the control execution as well as the data communication between control modules.

In this paper, we investigate several different architectures for a distributed control system for a reconfigurable machining system. We describe how the control requirements for a manufacturing control system map to temporal constraints on the data managed for the environment. We show how the various time delays associated with distributed architectures impact the performance of the control algorithms, and describe different types of communication protocols that can be implemented to meet the required deadlines.
\end{abstract}

\section{Introduction}

Currently, the commercially-available control systems for machine tools are not reconfigurable. Modular control systems are being investigated at several research locations, but to our knowledge, few address directly the coordination problems among control processes running in a distributed environment.

Although control theorists traditionally consider algorithm development, at some point the algorithms must be converted into software which can run on one or more computers. The analysis and design of distributed real-time control systems in a modular heterogeneous environment requires the study and integration of two complementary areas:

\footnotetext{
*This research was supported in part by the NSF Engineering Research Center for Reconfigurable Machining Systems at the University of Michigan.
} 
- development of modular, decentralized, reconfigurable real-time control strategies - i.e, an area within control systems.

- design and implementation of modular, distributed real-time coordination software, on heterogeneous platforms - i.e., an area under computer software systems.

Within this research area, we are looking at some of the issues in real-time and distributed implementation of control algorithms for machine tools. Once a modular control system has been designed, and the interaction between control modules specified, there are many different ways in which the control modules can be mapped to a hardware implementation. A multi-processor architecture is also modular from a hardware perspective, and can be more reconfigurable than a single-processor control system. However, the communication overhead associated with a multi-processor system may impact the control performance significantly. We are especially interested in evaluating the performance of decentralized control systems, in which there is no centralized scheduler, to increase modularity. As processors become more powerful and and networks become faster, this type of control architecture has the potential to be both high-performance as well as modular and reconfigurable.

In this paper, we investigate several different architectures for a control system for a reconfigurable machining system. The delays inherent in these different types of control systems are enumerated and their effects on the system performance evaluated. We show how the various time delays associated with distributed architectures impact the performance of the control algorithms, and describe different types of communication protocols that could be implemented to meet the required deadlines. In addition, a twoaxis contouring system is considered in some detail, and the effects of various delays on the contour error are determined by simulation. These analytic and simulation results can be used to specify the maximum allowable communication delays in the system. These control requirements for a manufacturing control system can be mapped to temporal constraints on the data managed for the environment.

\subsection{Reconfigurable machining systems}

The overall goal of the Engineering Research Center for Reconfigurable Machining Systems at the University of Michigan is to develop the necessary theory and technology to enable the next generation of machining systems to be quickly and easily reconfigured in response to changing market demands and new technology innovation [15]. One important facet of this vision is a reconfigurable control system. Such a system must be easy to design and must be composed of standard modules (hardware and software) that are interchangeable and extensible. It must be upgradable, so that new sensors and control algorithms can be added to existing systems.

Currently, most manufacturing systems are controlled using NCs, CNCs, and PLCs - which are relatively inflexible. The trend is toward using the more flexible PC-based systems for such control. Furthermore, to allow for modularity and distribution, the aim is to allow automated generation of, say, C code given the control specification for a particular configuration. At present, such automated code generation is primarily for centralized computing units.

In order to design and build modular controllers for modular machine tools, the issue of modularity itself must first be examined. It must be understood how different control algorithms should modularized (what is the appropriate granularity) and how they should be combined. In this paper, we assume that the modules are defined according to their purposes: position servo, cross-coupling, process control, etc. We consider the issues of how the control algorithm modules should be allocated among the many processors and how the communication between processors should be scheduled; we also discuss the performance tradeoffs associated with the different choices.

Decentralized control architectures are desirable for reconfigurable machining systems. Each machine module can have its own control module, complete with sensors, microprocessors, and a network connection. 
As the mechanical modules are fitted together to create a fully functional machine, so will the control modules be linked together over the network to create a fully functional control architecture. Although the decentralization has the advantages of simplicity and modularity, the communication delays over the network can lead to decreased stability and performance of the overall system.

\subsection{Machine tool control}

Our focus is on machining operations which are accomplished through the relative motion of a tool and the workpiece. We consider a contouring machining system as an example (though the techniques which we develop may be generalized). In contouring systems, the cutting occurs as the axes of motion are moved (e.g., as in a milling machine). The axes of motion may move simultaneously, each at a different velocity.

The two major performance criteria for machining operations which we will consider are productivity and accuracy. To achieve higher productivity (measured by the number of parts made over a given time period), the machine tool must move quickly with respect to the workpiece. This high feed-rate may decrease the accuracy of the produced parts. The most desirable balance between productivity and accuracy is chosen when the part program is written. Advanced control techniques which can increase the feed-rate without decreasing the accuracy are desirable, but due to the closed architecture, cannot be implemented within current commercially-available machine tool control systems.

The accuracy of a machine tool is often described in terms of the axis errors and the contour errors (i.e., various deviations from the precisely required trajectories). While in a machining system there are many sources of errors, in this work we focus on the errors associated with real-time, distributed communication and computation. We consider different controller architectures and the impact of these types of errors on the performance of the data and coordination of control system. Therefore, we assume that there are no errors associated with imperfect modeling of the axis motions, and no disturbances such as electrical noise, thermal deformation, or sensor inaccuracies associated with the machine.

\subsection{Data management}

The interactions between the control system and the manufacturing environment, involving large quantities of real-time sensor, actuator and control data, need to be managed correctly and efficiently. The states of the machine tools, the sensor readings, actuator signals, and control variables, all together represent the data to be managed. The various manufacturing activities that take place are coordinated in real-time, and in turn, they impose certain consistency and temporal constraints on the managed data. Yet, it is also necessary to allow autonomous executions in the distributed environment to allow for modularity and reconfigurability. Below, we describe the target domain in more detail in order to explain how the constraints arise in this practical domain.

The current approach to designing and developing machine control in manufacturing is a very laborintensive process. It usually involves developing all the application software, even though much of the code is similar to that written for other applications. The code execution is timed in an ad hoc manner, and experiments are run to check if the code can satisfy the real-time requirements of manufacturing. This software development process requires considerable effort, is very costly, and often takes several months. If the manufacturing requirements are changed, the code must be re-developed, and experiments done again to verify that the new code works correctly. Such issues are common in the development of real-time computing software (e.g., see [23]). To alleviate these problems in the context of reconfigurable manufacturing, we are investigating real-time data and coordination control strategies that are expected to run on commercially available computing platforms. Our research on the software for distributed coordination protocols has identified the coordination constraints in manufacturing systems that need support from a computing system. We propose imposing coordination constraints using a distributed database of the associated data, and 
associated temporal constraints among the data. Thereafter, we consider Quality of Service (QoS) issues in terms of meeting the real-time requirements for data management. The data includes sensor, actuator, and reference coordination data.

\subsection{Related work}

There are currently several related real-time control and coordination efforts at varying stages of development and deployment; a more detailed literature review can be found in [25].

\subsubsection{Machine-tool control architectures}

The Robotool is a 5-axis milling machine at University of Michigan with a first generation object-oriented open architecture controller [13]. This object-oriented modular programming architecture can simplify the writing of control programs. Robotool is a good attempt at creating modular controllers. There is some support for meeting real-time requirements, and some support for using multiple processors. However, the effort is not directed toward decentralized control of machine tools (the control is centrally scheduled), leaving unexamined most of the issues related to decentralized real-time control.

In the context of the Robotool, Koren et al. describe the effect of timing issues on an open-architecture machining system $[13,14]$. They consider the four basic types of control that must be performed on a single machine tool:

1. adaptive compensation and event management

2. interpolation

3. servo control

4. emergency control

and determine the minimum sampling time as the sum of the execution times of each type of control. Better performance can be achieved using a hierarchical controller with different sampling times for each of the three levels of control (this allows the servo control, the most critical, to execute more frequently). However, even in the hierarchical framework, it is difficult to reconfigure the control, because if a new axis were to be added, all the sample times must be recomputed. A multi-processor architecture could alleviate many of these problems; a real-time network would be necessary to allow communication amongst the various processors.

Altintaş et al. $[1,2]$ have proposed a modular design for a CNC. They use a hierarchical architecture with two buses: the main (ISA) bus and the CNC bus. On the main bus, there are several processor boards, each dedicated to a specific machine tool monitoring or control task (such as data acquisition, chatter detection and suppression, force control). The number of boards that can be added is constrained by the number of physically-available ISA slots. One of these processor boards is designated as the CNC master; it performs the interpolation as well as the real-time job management for the axis-level controllers. Manufacturing Automation Laboratories [19] has commercialized many of these technologies. The timing issues associated with this architecture have not been fully examined.

\subsubsection{Fieldbus-based systems}

Decotignie et al. describe an integrated communication and control system in [3]. They analyze the delays associated with communication over a fieldbus for both parallel and sequential computation at the distributed processors. The computations are assumed to be synchronized over the network with a unknown period, and a processor will only initiate a computation if all the necessary inputs from its predecessors are available. 
Both feedforward (interpolator) and feedback (servo) control strategies are considered, and the (perhaps obvious) conclusion is drawn that a higher number of axes can be handled if the fieldbus is placed between the interpolator and the axis control loops than if the axis control loops are closed over the fieldbus.

Song et al. also describe a distributed control architecture over a fieldbus [20]. They describe a multiaxis machine-tool with one processor for the central control (in their example, interpolation) plus another processor for each axis servo control. Synchronization between axes is done by time-synchronizing the axis commands over the real-time network.

They describe the distribution of a control system by three steps: the functional architecture, the hardware architecture (processors and networks), and the operational architecture (which is mapping the functional definition onto the chosen hardware). They briefly describe the Structured Analysis and Design Technique which breaks the functionality down into a set of elementary data processing modules called distribution atoms which can neither be distributed on several nodes nor partly executed. However, they note that the description formalism is not yet fully defined. Also, no formal methods exist for optimally distributing these atoms onto a hardware architecture.

Ferrarini et al. [4] have looked at hybrid position and force control for an industrial robot; they enable force control when contact is made, and turn it off when contact is broken. Their control is implemented in a distributed environment. In essence, they have reconfigured their system by adding an extra CPU board to an existing architecture to handle the added force control functions.

\subsubsection{Other real-time control efforts}

Onika is an on-going project at Carnegie-Mellon University which was built to implement real-time controllers for robots [6]. Onika has a multilevel human-machine interface for real-time sensor-based systems. The upperlevel provides a user-friendly interface to combine different control objects. The middle-level has the actual code associated with the control objects. Finally, the device drivers and sensor interfaces are in the lowest level. These layers are built on top of a proprietary real-time operating system. Control modules execute on multiple processors, and communication between them is managed through shared memory. However, it does not address real-time communication between control modules which do not share a common bus. It has little support for Quality of Service trade-offs between quality and performance.

Wittenmark et al. [27] give a brief overview of some timing problems in real-time control systems. They enumerate three types of problems: control delay (the elapsed time between sensing and actuation), jitter (time-variation in start time of actions), and transient errors (due to loss or corruption of control-related signals during communication). They show, through simulation, how delays can affect performance. The inverse problem, that of specifying the maximum allowable delay given a specified performance criteria, remains unsolved.

\section{Machine tool control structure}

Following Koren et al. [13], in this paper we consider three basic types of control modules for a multi-axis machine tool controller: servo, interpolation, and process. Emergency control will not be considered here.

\subsection{Servo control}

First we consider the axis-level controllers, also called servo controllers. In an effort to simplify the problem, in order to highlight the effects of communication and computation delays, we model each axis of a machine tool as a simple second-order transfer function $P(s)$.

$$
P(s)=\frac{X(s)}{V(s)}=\frac{K}{s(s+a)}
$$


More precise models can be found in [11, 12, 8].

Typically, each axis will have its own servo controller to allow it to track reference inputs. Servo controller operates in discrete-time with a sampling time of $T_{s}$. A proportional-integral (PI) control law gives a closedloop axis system of Type II, allowing ramp reference inputs to be tracked with zero steady-state error. The continuous-time transfer function of our PI controller is given by

$$
C(s)=K_{P}+\frac{K_{I}}{s}
$$

and a discrete approximation to this continuous-time controller is constructed using the bilinear approximation [5].

It has been shown that a slightly more complicated PID controller can improve the performance of the system [17], although most industrial machine tool controllers use the simpler PI algorithm [26]. Again, we have chosen to use simple models for our preliminary study to highlight the effects of the delays in the system. One potential reconfiguration of a machine tool system would be to upgrade some (or all) of the servo controllers from PD to PID. Another potential upgrade would be to add friction compensation to the servo-level controllers to decrease the errors due to stiction effects $[7,8]$.

\subsection{Interpolation}

An interpolator coordinates the axes by decomposing the desired operation motions into individual axis reference commands [11]. The input to this module depends on the type of motion (such as linear, circular, or parabolic). However, the most basic inputs to this module are the initial and final positions of each axis and the desired feed-rate (the speed of the tool relative to the workpiece). Using this information, the interpolator generates the reference positions for each axis. In conventional machining operations, the desired feed-rate for each motion is stored in the part program. Intelligent machining systems often adjust the feed-rate in real-time to increase operation productivity and quality using adaptive process control (as described in the following section).

\subsection{Adaptive process control}

Adaptive or process control can be used to optimize productivity and accuracy [16]. Even though there are numerous types of process control, most of them are implemented by adapting the desired feed-rate and/or positions in response to external inputs or disturbances. For example, force control changes the feed-rate to maintain a constant force on the tool; this can prevent tools from breaking and minimize tool wear. Chatter suppression can be done by either decreasing the spindle rotational speed or decreasing the depth of cut; newer results show that varying the spindle speed sinusoidally can also help to reduce chatter [28]. Thermal and geometric compensators change the initial and final value to the interpolator to account for the thermal expansion or known geometric inaccuracies in the machine tool.

Adaptive control algorithms may operate at a different sampling time than the servo controls. Because they act by modifying the reference inputs to the servo controller (either directly or indirectly through the interpolator), this sample time is usually longer than the sample time of the servo controller but is never shorter (it may be the same). In contrast, the computation time associated with an adaptive control algorithm is often longer than that associated with a servo controller.

Adaptive control algorithms may require the knowledge of many sensor inputs (such as positions, forces, temperatures, etc.). This data may be transmitted over a network, causing communication delays which can affect the performance of the adaptive control algorithms. 


\subsection{Cross-coupled control}

A cross-coupled controller can improve the contouring accuracy (independent of the tracking accuracy of each axis) in a two-axis machine tool system [10,22]. The cross-coupled control module coordinates the motion of the two axes to minimize the contour error. It takes as inputs both the $x$ and $y$ references and sensed positions, and computes the distance between the actual $(x, y)$ position and the desired contour. It uses this difference to either compute appropriate control voltages for both the $x$ and $y$ axes to add to the outputs of the servo controllers, or to send a signal to the interpolator which then modifies the reference values to the servo controllers. Thus, a cross-coupled controller can be considered either as part of the servo loop (if its action is to modify the commanded voltages to the motors) or as part of the adaptive process control (if its action is to modify the commanded references to the servos).

There are two major parts in the cross-coupled control algorithm: the estimation of the contour error and the design of cross-coupled compensator. To estimate the contour error, the cross-coupled control algorithm needs measurements of the actual position of $x$ and $y$ as well as the reference inputs. The number of data points needed from each subsystem is determined by the desired accuracy of the contour error estimation. The contour error can be estimated by [22]

$$
\begin{aligned}
\varepsilon_{x} & =\left(\sin ^{2} \theta\right) e_{x}-(\cos \theta \sin \theta) e_{y} \\
\varepsilon_{y} & =\left(\cos ^{2} \theta\right) e_{y}-(\cos \theta \sin \theta) e_{x}
\end{aligned}
$$

where $\theta$ is the instantaneous inclination of the tangent to the contour path. For the cross-coupled compensator, a simple gain controller will suffice $[10,22]$ although more complex control laws may give even better performance.

The steady state value of the contour error is highly dependent upon the overall gain of the cross-coupling controller. Larger values of the cross-coupled gain result in decreasing contour error; however, if the gain is too high, the system can become unstable (as shown in Section 4.4).

\subsection{Example: A two-axis contouring system}

The main example that we will use in this paper is a simple two-axis milling machine for contouring applications. The milling machine has a vertical ( $z$-axis) spindle on which the tool is mounted, and an $x-y$ table to position the metal underneath the mill. For simplicity, we assume that the position of the spindle is fixed; we only consider the motion of the $x-y$ table. For this example, we will examine the effects of delays on the performance of a servo and cross-coupled control system; process control algorithms are currently under investigation.

Let the $x-y$ table trajectories be $\left(r_{x}(t), r_{y}(t)\right)$, and the actual sensed positions be $(x(t), y(t))$. We assume that the reference trajectory is a circle with center $\left(x_{c}, y_{c}\right)$ and radius $R$, hence:

$$
\left(r_{x}(t), r_{y}(t)\right)=\left(x_{c}+R \sin (t), y_{c}+R \cos (t)\right)
$$

In a contouring system, the control performance criterion is given by the contour error. The contour error $(\varepsilon)$ is defined as the shortest distance from $(x, y)$ to the desired circle:

$$
\varepsilon(t)=\sqrt{\left(x(t)-x_{c}\right)^{2}+\left(y(t)-y_{c}\right)^{2}}-R
$$

which is not the same as $\sqrt{e_{x}^{2}+e_{y}^{2}}$.

We will use a cross-coupled controller which estimates the contour error using equations (3)-(4) and compensates for it using a constant gain $W$ (we assume $W_{x}=W_{y}=W$ ). 


\section{Computing and communication architectures for control}

The performance of a distributed control system is determined not only by the control algorithm but also by the computing and communication architecture with which the algorithm is implemented. Both computation and communication delays occur in a distributed system, and their effects must be considered. In general, a given control algorithm could be implemented in many different ways, with different numbers of processors and different communication networks.

In this section, we define four different architectures for machine tool control and examine the advantages and disadvantages of each architecture in terms of reconfigurability and expected performance. For each of the several different types of architectures, we will discuss the types of delays that occur. The effect of these delays on the transient and steady-state performance of the system will be analyzed in the following section. Because we are interested in designing control systems for reconfigurable manufacturing systems, we also briefly discuss the ease with which different configurations can be reconfigured or upgraded on an as-needed basis.

\subsection{Sources of delay}

The two main sources of delay in a control system are the communication delay associated with sending information over a network, and the computation delay associated with computing control algorithms on a microprocessor. In Figure 1, we have shown a simple block diagram of a control system and noted several locations where time delays can occur. A delay in between the servo controller and the plant is caused by the computation time needed to compute the servo control algorithm and is denoted $T_{C P}$. A sensing delay from the output of the plant back to the servo controller is denoted $T_{P C}$. A delay between the interpolator and the reference input to the servo controller, due to communication delays, is denoted $T_{R C}$. In the event that adaptive process control is contained in the loop, there could be sensing delays to the controller $T_{P A}$ as well as communication and computation delays between the adaptive control block and the interpolator, which are lumped together as $T_{A R}$. Even though there will be only one sensor per state, two different delays $\left(T_{P C}\right.$ and $\left.T_{P A}\right)$ are shown in Figure 1 to represent the fact that the time required for the sensed data to reach the different control blocks depends on both the network and the system architecture.

\subsection{Centralized architecture}

We define a centralized control architecture as a single block which computes the control commands to all the actuators of the system and has complete knowledge of the state of the entire system. It is typically designed around a complete multi-variable model of the plant, and uses the position sensor data from all axes. This large block may also contain the interpolator, or it may compute the reference values separately.

A conventional multi-variable controller would be one example of an architecture which fits into this category. Since a centralized control algorithm has complete knowledge of the system, and can compensate for coupling between axes through the multi-variable system model, it has the best possible performance of all the control design architectures. In order to achieve this level of performance, however, the centralized controller must be implemented on a single processor with negligible computation and sensing delays. This is certainly possible for systems with one or two axes, but becomes more difficult for many degree-of-freedom machines. In addition, as the number of axes (and states) increases, the modeling and control design procedures become more complex. More complicated control algorithms are needed, which may increase the computational delay to unacceptable levels.

A centralized control architecture is not considered to be reconfigurable. If the machine is updated to have extra (or fewer) axes, or if a change in the control algorithm is desired, the entire system model must 


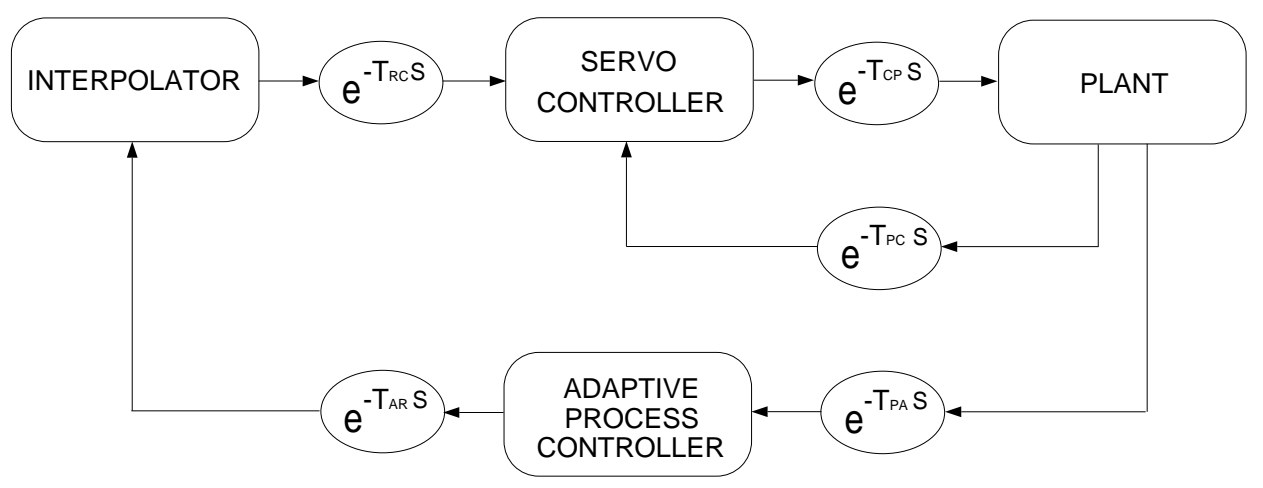

Figure 1: A simple two-loop control system with a servo controller, interpolator, and adaptive process controller, showing the possible delays in the loops. A delay in between the servo controller and the plant, caused by the time needed to compute the servo control algorithm, is denoted $T_{C P}$. A sensing delay from the output of the plant back to the servo controller is denoted $T_{P C}$. The delay between the interpolator and the reference input to the servo controller, due to communication delays on the network, is denoted $T_{R C}$. In the event that adaptive process control is contained in the loop, there could be sensing delays to the controller $T_{P A}$ as well as communication and computation delays between the adaptive control block and the interpolator, which are lumped together as $T_{A R}$.

be rebuilt and the controller redesigned. Although some automated techniques exist to assist in these steps, the process is still time-consuming and hence expensive.

Although a centralized control architecture is usually implemented on a single processor, a multi-processor system with a centralized scheduler may be implemented if the control is too complex for the available hardware to handle in the required sample time. We still consider this architecture to be centralized and not reconfigurable.

\subsection{Decoupled architecture}

The other extreme from a centralized architecture is a decoupled architecture. We define a control system to be decoupled if each actuated degree of freedom has its own control block with only local knowledge of the sensed outputs. For example, if each actuated axis has a sensor and a servo controller, but there is no communication among the servos, the control system would be called decoupled. As the name implies, a decoupled control architecture cannot compensate for coupling among the degrees of freedom. In a machining application, there will still be a central interpolator which generates the desired trajectories for all axes.

Although a decoupled control architecture could be implemented on a single processor, the structure naturally lends itself to distribution amongst many processors. This type of distributed multi-processor structure can decrease the magnitude of both the communication and computation delays. For example, if each axis has its own processor and sensor, and the only tasks that the processor must perform are reading the sensor value and computing the servo algorithm, the time delays involved will be relatively small. There will still be a network connection to communicate the reference value for the servo controller, but the sensor data does not need to be communicated across any sort of network. A single-axis servo control algorithm is typically quite simple and does not require extensive computation time.

Because of the minimal communication and computation delays, a decoupled control architecture can support a small servo-level sampling time, potentially increasing the accuracy of the axis-level positioning. Such a system may still have significant contour errors. In addition, a decoupled control architecture is easily reconfigured; when an axis is added to or removed from the system, only the interpolator needs to be 
reprogrammed.

\subsection{Hierarchical architecture}

A hierarchical architecture has some of the advantages of both the centralized and decoupled architectures. We define a control system to be hierarchical if each axis has its own servo level controller (with only local state knowledge) and there also exists a supervisory controller with knowledge of the state of the entire system. This supervisory block might be an adaptive process controller, a cross-coupling controller, or a combination thereof. The sensor data may be sampled locally, at the servo level, and communicated up to the supervisory block over a network; it may be sampled at the supervisory level and sent to the servo level over a network; or there may be some combination of these two extremes (such as, position measurements taken locally at the servo but force measurements taken at the supervisory level). The communication delays of sensed data to each control algorithm will be different in the two cases. The computation delay of the servo controller is typically small, but the supervisory controller is often more complex and may have a significant computation delay.

Because it has global knowledge of the complete state of the system, and can compensate for coupling among the axes through the supervisory control algorithm, a well-designed hierarchical architecture can always out-perform a decoupled one. Furthermore, unlike the centralized architecture, the hierarchical architecture allows the usage of a distributed, multi-processor structure, which can increase the reconfigurability of system significantly. If the system is reconfigured to have more or fewer axes, only the supervisory control algorithm needs to be reprogrammed and reverified. In addition, modification of the servo control algorithm would not affect the supervisory controller, and the only timing issues that need to be examined with such a change are those of the servo-level controller. Within such an architecture, however, the communication delay could increase to unacceptable levels because the sensed data always have to travel over a network.

\subsection{Decentralized architecture}

A control architecture is defined to be decentralized if the control block associated with each servo axis (or group of axes) has knowledge of some (or all) remote states (in addition to all local states). There is no 'supervisory' control block with global knowledge of the system. Data is communicated between the control blocks over a network. The interpolator still coordinates the many degrees of freedom by sending the desired reference positions over the network. Adaptive process control or cross-coupling control can be included in this architecture by adding another control block connected to the network or by incorporating part of the control algorithm in each servo-level block.

This type of system architecture has the potential to be easily reconfigurable. As in the hierarchical framework, the servo controllers can be changed or updated without affecting the global framework. Also, this architecture has the potential for intelligent self-reconfiguration in the following sense. Each servo-level control block could activate the appropriate locally-stored control algorithms (such as cross-coupled or force control) depending on the availability of the relevant data on the network. When an axis is added to or removed from the machine, the controls associated with the other axes will automatically compensate for the change. The interpolator may still need to be reprogrammed.

\subsection{Architectures for the two-axis contouring example}

In this section, we describe the different architectures we consider for the two-axis system introduced in Section 2.5. Although for our two-axis system the control modules we are considering are very simple and would almost always be implemented on a single processor, we consider the four different proposed 
2 axis system with cross coupled controller

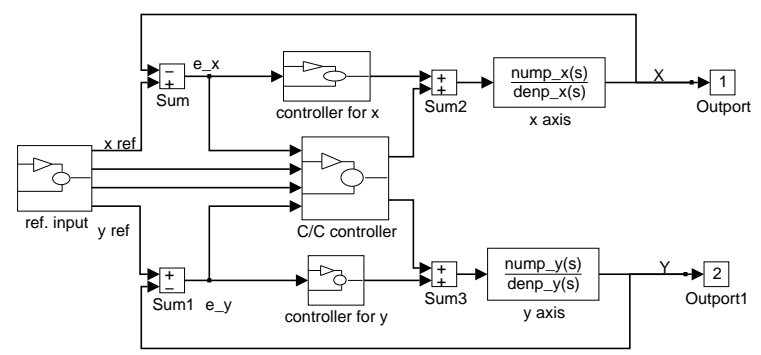

a. Hierarchical

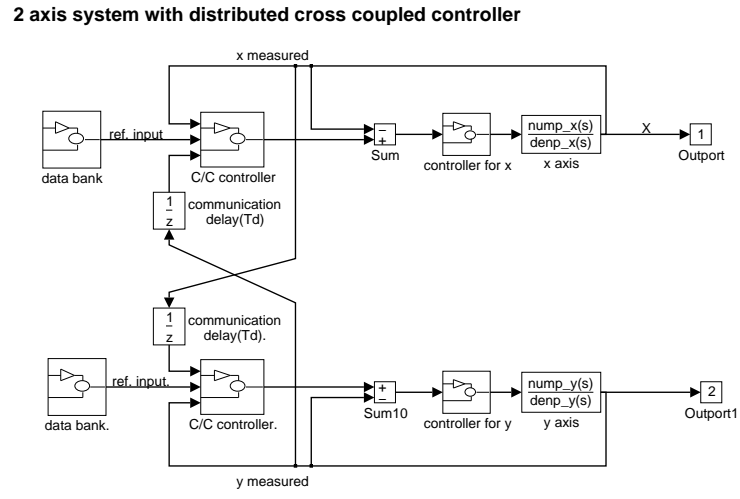

b. Distributed

Figure 2: Simulink diagram of the cross-coupled controller. In each configuration, each axis has a servocontrol module which is a simple PI controller. For the hierarchical implementation, there is a cross-coupling module which coordinates the motion of the two axes. For the distributed implementation, there is a crosscoupled control module for each axis.

architectures in order to study the communication requirements of the different types of controllers and the effects of communication and computation delays on the control performance.

We consider four basic control architectures for the two-axis milling machine example; these are shown in Table 1. The two extremes are a centralized controller, in which there is no distributed processing, and a decoupled controller, in which there is no communication between the two axes. The decoupled controller is the simplest type of control architecture, consisting only of the two servo-level control modules, but has the worst performance since the contour error is not considered and thus cannot be compensated. As noted above, the performance of a machine tool control system can be improved by using a cross-coupled controller. The same cross-coupled control algorithm can be implemented in two different ways: as a hierarchical controller, or in a decentralized (but coordinated) manner.

The control structure shown in Figure $2 \mathrm{a}$ is considered to be modular as compared to a centralized controller (the first block in Table 1). There is a servo control module for each axis $(x$ and $y$ ) in addition to the cross-coupling module. We also consider the cross-coupled controller in a distributed architecture as shown in Figure 2b. The distributed control increases the modularity of the system by having a cross-coupling control module for each axis but does not change the nominal system performance (i.e., the control equations remain unchanged). Different types of time delays will affect the system in each type of architecture; the effects of these delays will be shown in Section 4.4 .

\section{Effects of computation and communication delays}

In order to determine the best control system architecture for a reconfigurable machining system, we must first understand the effects of communication and computation delays associated with the different architectures. It is commonly known that delays in a control system tend to decrease performance and can even cause instability. Due to the nonlinearity associated with time delays, however, it may be difficult or even impossible to solve analytically for the effects of delays. In addition, if the system is reconfigured, and the control structure or algorithms change, the analysis should be done again.

In this section, we present a combination of simulations and analysis to show how time delays can affect the system's performance. As might be expected, different types of time delays have different effects. Time 


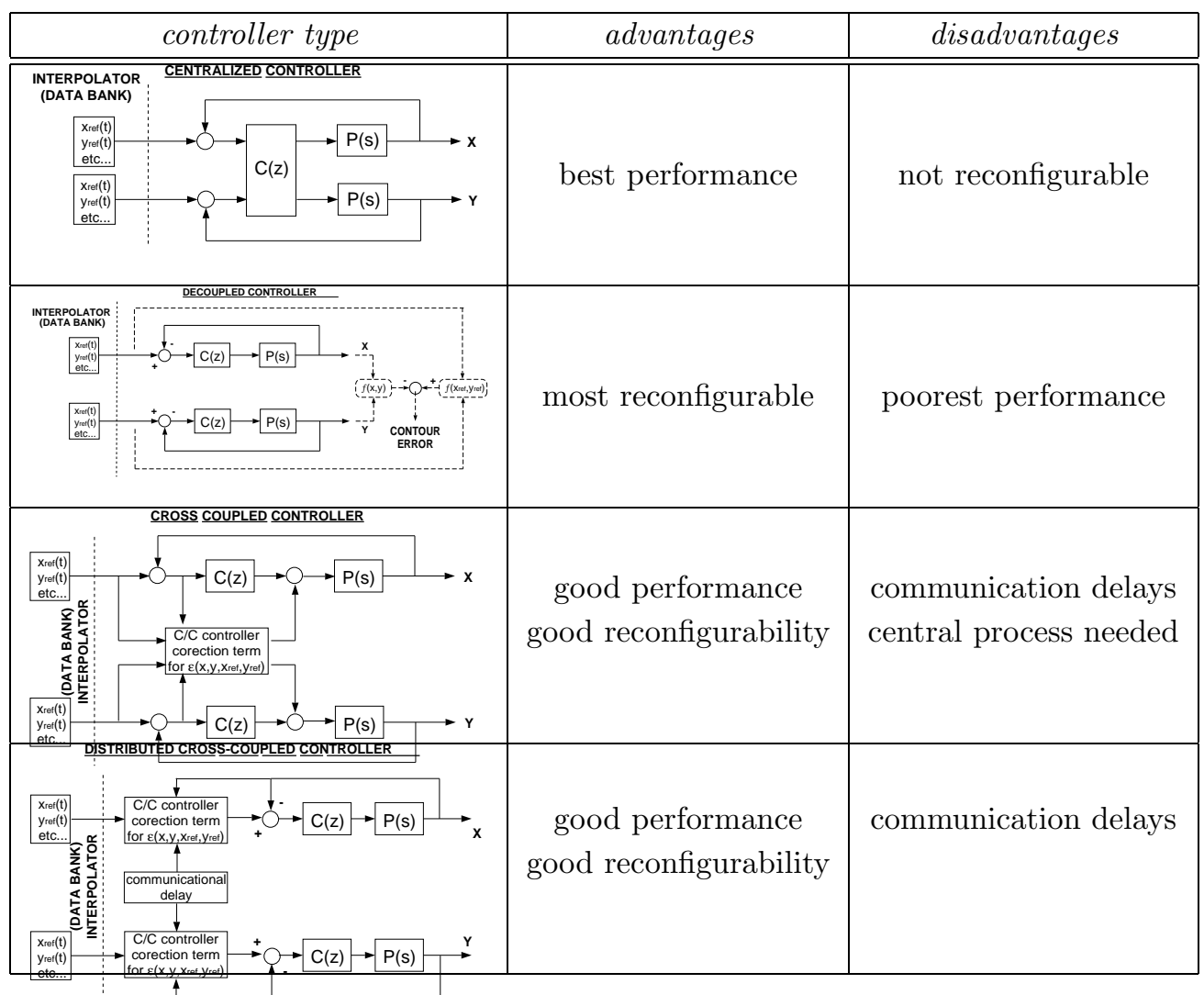

Table 1: Four different control architectures, and comparisons, for a two-axis milling machine: centralized, decoupled, hierarchical cross-coupled, and decentralized cross-coupled . 


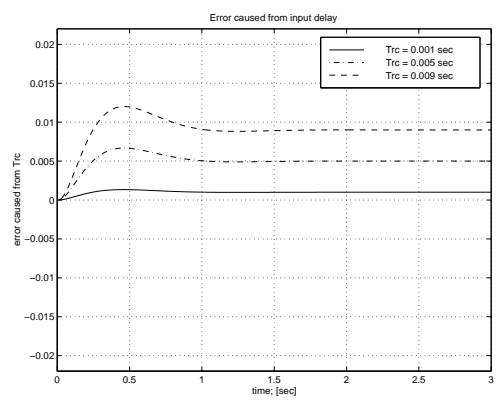

a. reference delay $T_{R C}$

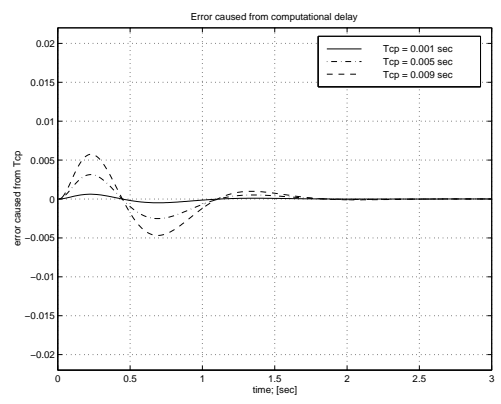

b. computational delay $T_{C P}$

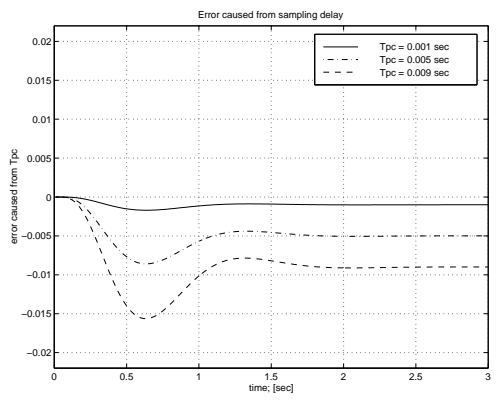

b. sensing delay $T_{P C}$

Figure 3: Errors caused by time delays, comparing the effects of magnitude and location. The reference delay $T_{R C}$ from the interpolator to servo controller, the computation delay $T_{C P}$ in the servo controller, and the sensing delay $T_{P C}$ between the sensed outputs of the plant and the servo controller are considered. For each type of delay, three separate magnitudes $(0.001,0.005$, and $0.009 \mathrm{sec})$ are considered. The sampling time $T_{s}$ of both the servo controller and the interpolator is $0.01 \mathrm{sec}$. The errors are expressed in terms of the difference between the outputs of the system with time delay and the system without delay; the input to the system is a ramp with unit slope. Note that the maximum and the steady state errors depend not only on the size of the delay but also the location of the delay.

delay tends to increase the maximum error as well as the average error, and may also change the steady state error of system. We will also show how certain combinations of time delays can actually result in improved performance over single time delays. We expect that this type of simulation and analytic information will be useful not only in choosing the best type of system architecture, but also in designing the communication protocols that will be used in a reconfigurable machining system (as described in Section 5).

For this preliminary study, we have considered a single-axis, single-loop servo system with an interpolator. We analyzed the effects of the time delays shown in Figure 1 when the servo controller is a PI algorithm, the plant is a second-order motor, as described in Section 2.1, and the reference is a ramp function with unit slope.

The errors that we present in Figures 3 and 4 are all expressed as compared to the same system without any time delay. As shown in Figure 3, the effect of the time delay depends not only on the magnitude of the time delay but also on its position in the control loop. The effects of the combination of different types of time delays in the system are shown in Figure 4. Of particular interest is the fact that for this simple system, the errors due to the time delays are additive. Most notably, the positive and negative steady-state errors associated with the reference and sensing delays cancel. This indicates that if one of these types of delays is unavoidably present in the system, the system designer may compensate for it (at least in the steady-state) by intentionally introducing a delay on the other.

After we have described the effects of time delay in the single-loop system, we consider the two-axis contouring system with cross-coupling control as described in Section 2.5. For this example, the closed-loop equations are nonlinear, and thus we have not done any analytic computations. In Section 4.4, the effects of the sampling time of the two different control algorithms (servo and cross-coupled) as well as the effect due to time delays in various parts of the loop will be discussed. The performance criterion for this system is the contour error.

\subsection{Analytic results}

The modified $\mathcal{Z}$-transform is an extension of the traditional $\mathcal{Z}$ transform which is useful in the study of combined differential and difference equations arising in the study of both hybrid (mixed continuous- and 


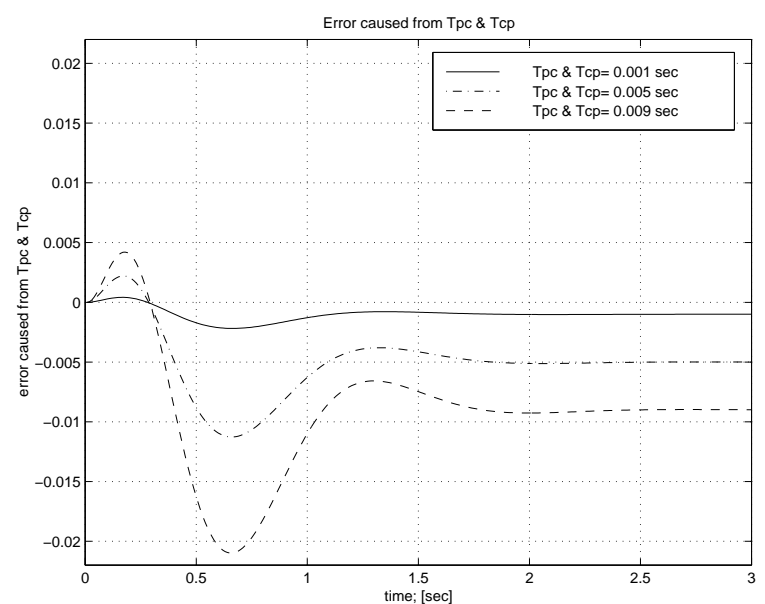

a. Computation delay $T_{C P}$ and sensing delay $T_{P C}$

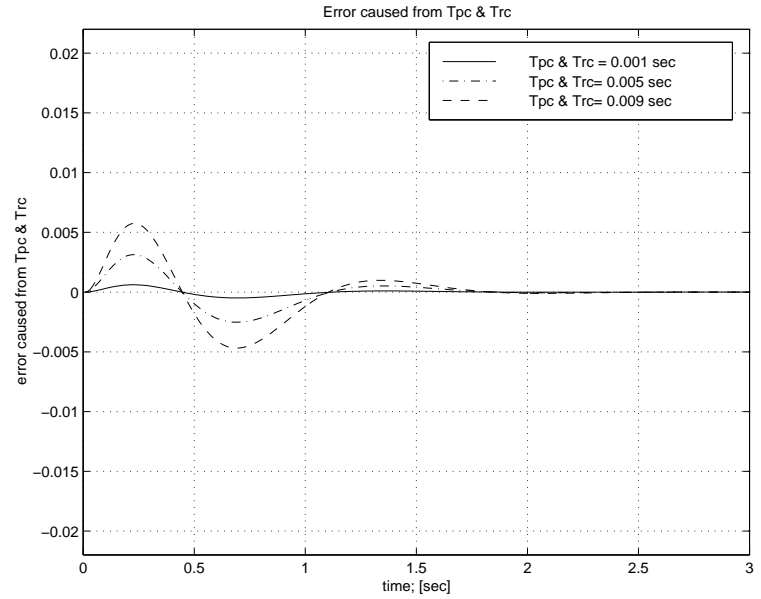

c. Sensing delay $T_{P C}$ and reference delay $T_{R C}$

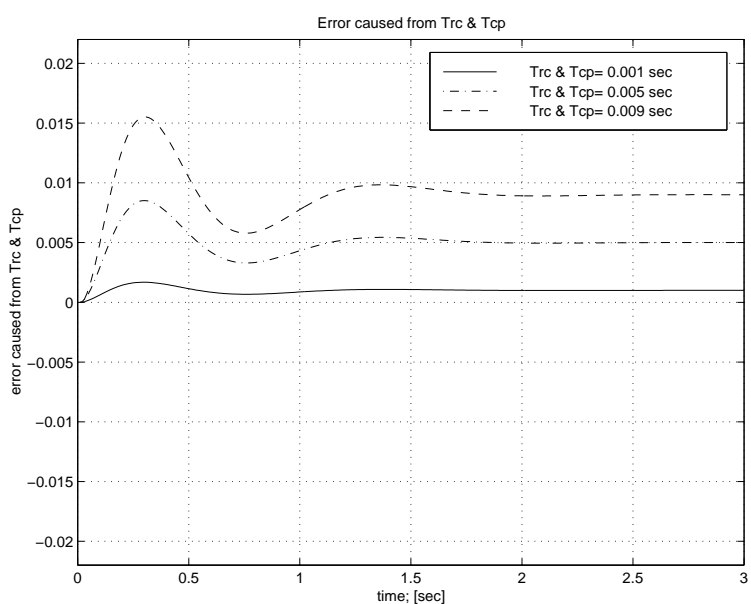

b. Computation delay $T_{C P}$ and reference delay $T_{R C}$

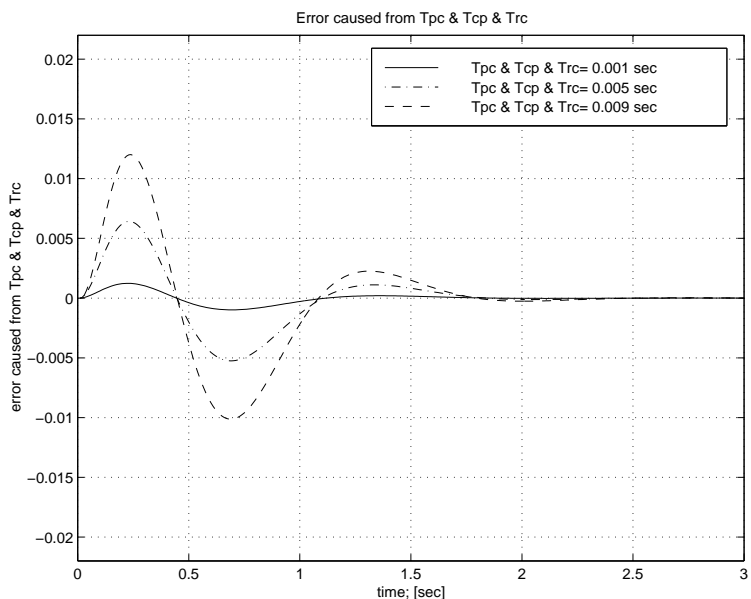

d. Computation $T_{C P}$, sensing $T_{P C}$, and reference $T_{R C}$ delays

Figure 4: Here we show the effects of different combinations of delays. In each figure, three different magnitudes of delay are considered $(0.001,0.005$, and $0.009 \mathrm{sec})$; the same magnitude is used for each type of delay. The sampling time of the servo controller in all cases is $T_{s}=0.01 \mathrm{sec}$. The errors are expressed in terms of the difference between the outputs of the system with time delay and the system without delay; the input to the system is a ramp with unit slope. Of particular interest is the fact that for this simple system, the errors due to the time delays are additive. Most notably, the positive and negative steady-state errors associated with the reference and sensing delays cancel. This indicates that if one of these types of delays is unavoidably present in the system, the system designer may compensate for it (at least in the steady-state) by intentionally introducing a delay on the other. 
discrete-time) systems and time delay systems. This analytic approach can be applied to obtain the value of a function $f(t)$ between the sample times as well as to compute the discrete transfer function of a continuous system with time delay [5, 18]. Using this method, possible analytical error introduced by other methods (such as the Padé approximation) can be eliminated. Furthermore, the modified $\mathcal{Z}$-transform has the desirable properties of the standard $\mathcal{Z}$-transform such as linearity, final value theorem, etc., greatly simplifying the analysis of the system [18]. In this section, we describe how the modified $\mathcal{Z}$-transform can be used to analyze the closed-loop behavior of our example system with time delays.

Let the plant be a second-order type I system with transfer function

$$
P(s)=\frac{K}{s(s+a)}
$$

For the examples we consider in this paper, this transfer function represents the motor of the machine tool as described in Section 2.1. The system with time delay $\lambda$ can be expressed as

$$
P^{\prime}(s)=e^{-\lambda s} P(s)
$$

We will use the modified $\mathcal{Z}$-transform to compute the exact output of the system at each sample time. Let the delay $\lambda$ be expressed in terms of the sampling period as

$$
\lambda=(\ell-m) \cdot T_{s}
$$

where $T_{s}$ is the sampling period, $\ell$ is an integer and $m$ is a nonnegative number less than 1 . If the delay is an exact multiple of the sample time, then $m=0$.

The discrete transfer function $P^{\prime}(z)$ of $P^{\prime}(s)$ can be computed as follows. The main idea is to directly take the $\mathcal{Z}$-transform of the integral number of time delays $(\ell)$ and then compute the time-shifts in the step and exponential functions due to the fractional time-delay $(m)$.

$$
\begin{aligned}
P^{\prime}(z) & =\frac{z-1}{z} \mathcal{Z}\left\{e^{-(\ell-m) T_{s} s} \cdot \frac{P(s)}{s}\right\} \\
& =\frac{z-1}{z^{\ell+1}} \mathcal{Z}\left\{e^{-m s} \cdot \frac{K}{s^{2}(s+a)}\right\} \\
& =\frac{K}{a^{2}} \frac{z-1}{z^{\ell+1}} \mathcal{Z}\left\{a\left(t+m T_{s}\right)-1+e^{-a\left(t-m T_{s}\right)}\right\} \\
& =\frac{K}{a^{2}} \frac{z-1}{z^{\ell+1}}\left(\left(a m T_{s}-1\right) \frac{z}{z-1}+\frac{a T z}{(z-1)^{2}}+\frac{e^{-a m T_{s}}}{z-e^{-a T_{s}}}\right) \\
& =\frac{K}{a^{2}} \frac{n_{1} z^{2}+n_{2} z+n_{3}}{z^{\ell}\left(z^{2}-\left(1+e^{-a T_{s}}\right) z+e^{-a T_{s}}\right)}
\end{aligned}
$$

where

$$
\begin{aligned}
& n_{1}=a m T_{s}-1+e^{a m T_{s}} \\
& n_{2}=\left(1-a m T_{s}\right)\left(1+e^{-a T_{s}}\right)+a T_{s}-2 e^{a m T_{s}} \\
& n_{3}=\left(a m T_{s}-1\right) e^{-a T_{s}}-a T_{s} e^{-a T_{s}}+e^{a m T_{s}}
\end{aligned}
$$

This discrete transfer function $P^{\prime}(z)$, used with the inverse of the modified $\mathcal{Z}$-transform of the controller, enables us to find the error profile of the delay system [18].

\subsection{Communication delays}

We first consider the different types of communication delays that may be present in a system. The magnitude of the delays will depend not only on the chosen architecture but also on the speed and configuration of the 
chosen network. Some networks, such as Sercos, have fixed cycle times and deterministic operation. Others, such as Ethernet, are fast but can result in varying communication times. At present, our analysis only includes fixed delay times; the effects of varying time-delays will be considered in future work.

\subsubsection{Reference input delays}

In any type of distributed system architecture (decoupled, hierarchical, or decentralized), the interpolator will send the reference values to the servo controllers over a network, resulting in a time delay. If the time delay to all servos is the same, then the interpolator may operate in a "look-ahead" fashion to eliminate the resulting errors. More complex compensation may be required when there are differing time delays to different axes, or the time delay may vary due to a nondeterministic network.

Consider a servo system as described in Section 2.1 with a ramp reference. A communication delay $T_{R C}$ between the interpolator and the servo controller will result in a steady state error of

$$
e_{s s}=V_{r} T_{R C}
$$

Because the reference data reaches the servo controller after a delay of $T_{R C}$, the steady-state error due to the delay is equal to the reference velocity $V_{r}$ times the time delay $T_{R C}$.

This analysis holds true for a type I system with a PI servo controller (the closed-loop system is type II) and a ramp reference input with a slope of $V_{r}$. If the reference input were a step, then the steady-state error for the same controller and plant system would be zero for a reference input delay.

\subsubsection{Sensing delays}

Some or all of the sensed data may also be sent over a network. Some outputs (such as positions) are sampled at the highest frequency present in the system (usually the sample time of the servo loop). The minimum achievable sensing delay will depend on the amount of data that needs to be sent over the network (how many outputs are sampled during each period), how often the data needs to be sent (the sample time), as well as the bandwidth of the network. Of course, the network protocols and architecture will also influence the amount of delay.

Using the final value theorem, we determine that a sensing delay $T_{P C}$ will result in a steady state error of

$$
e_{s s}=-V_{r} T_{P C}
$$

This result is analogous to the error caused by reference input delay. Because here the system output is delayed in amount of $T_{P C}$, the actual output will end up leading the reference, giving a negative error.

Again, we note that for the simple system we have considered, the effects of the sensing and reference delays are additive. That is, the positive and negative steady-state errors associated with the reference and sensing delays cancel. This indicates that if one of these types of delays is unavoidably present in the system, the system designer may compensate for it (at least in the steady-state) by intentionally introducing a delay on the other.

Once again, this analysis holds true for a type I system with a PI servo controller (the closed-loop system is type II) and a ramp reference input with a slope of $V_{r}$. If the reference input were a step, then the steady-state error for the same controller and plant system would be zero for a reference input delay.

\subsection{Computation delays}

We also consider the delays associate with the finite computation time of the digital computer. We denote this computation time as $T_{C P}$. This delay depends not only on the complexity of control algorithm but also on the number of tasks a processor must complete during each sampling period. The effects of this delay 
can be used to determine the number of processors that should be used in the system in order to meet a given error specification. The control system designer should choose to allocate the tasks among multiple processors in the system in such a way as to minimize the effect of the computation delays.

The steady state value of the error caused by the computation delay is zero for the given system, even though the maximum overshoot error was increased as the magnitude of this delay increased (see Figure 3). In addition, the maximum overshoot error caused by this delay was less than the error from the communication delays $T_{P C}$ or $T_{C P}$. Thus, we should choose the control architecture of the system in order to minimize the communication delays as much as possible, even if that means increasing the computation delay by performing many tasks on a single processor.

\subsection{Simulation results for the two-axis contouring system}

We simulated the cross-coupled controller with a circular reference input and several different control frequencies and communication frequencies. We varied both the frequency of the servo-level controllers $T_{s}$ as well as the frequency of the cross-coupled controller $T_{c}$. We also considered the effect of communication delays on the cross-coupled controller. We defined the performance measure of our control system as the maximum contour error and we computed this measure for several different scenarios.

The cross-coupled controller for the two-axis machine tool system was shown in Figure 2. Using block diagram reduction, the cross-coupled controller can be implemented in a decentralized fashion; this increases the modularity of the system without attenuating the nominal performance of the controller. Both the hierarchical and decentralized architectures are shown in the figure.

The results of our simulations are summarized in Figures 5-7. As shown in Figure 5, the maximum contour error is an exponential function of the sampling time $T_{s}$. The frequency of the cross-coupled controller $T_{c}$ also affects the contour error, but its impact is highly dependent on the sampling time, $T_{s}$. For small sampling frequencies $\left(T_{s}<0.03 \mathrm{sec}\right)$, the sampling frequency of the cross-coupled controller less than four times the sampling frequency have no significant effect on the contour error. However, the sampling frequency of $\mathrm{C} / \mathrm{C}$ would dramatically increase the contour error as $T_{s}$ is increased, its effects are much more significant than the delay associated with the communication. The effect of the communication delay between axes also depends strongly on the sampling time $T_{s}$ (see Figure 6). In addition, the contour error depends strongly on the cross-coupled gain $W$ as shown in Figure 7.

We expect that simulation results such as these could be used as follows. For a given performance requirement (such as maximum contour error), the sample times of the servo-level control $T_{s}$ and the crosscoupled control $T_{c}$ can be specified from the figures. The maximum allowable communication delay can also be determined in a similar manner. The priority order of jobs can be specified as the following: first is necessarily sampling and computing the servo-level control for each axis, then sampling and computing the cross-coupled control algorithm for each axis, and finally data communication. In this manner, overrequirements on each task can be avoided, and if it is not possible to complete all tasks within a given time interval, the priority ordering is clear.

The sample times and communication requirements of the control algorithms will dictate the type and amount of computer hardware that will be required in the system, as well as the database structure and communication protocols that will be needed. Again, we emphasize that for this simple two-axis example, the performance requirements can be easily met with existing tools on a single processor. Machine tool performance requirements continue to become more stringent, with very high speeds and very high (micronlevel) accuracies. The next generation of machine tool controllers will require distributed computation and communication to achieve both the performance requirements and the desired reconfigurability. 


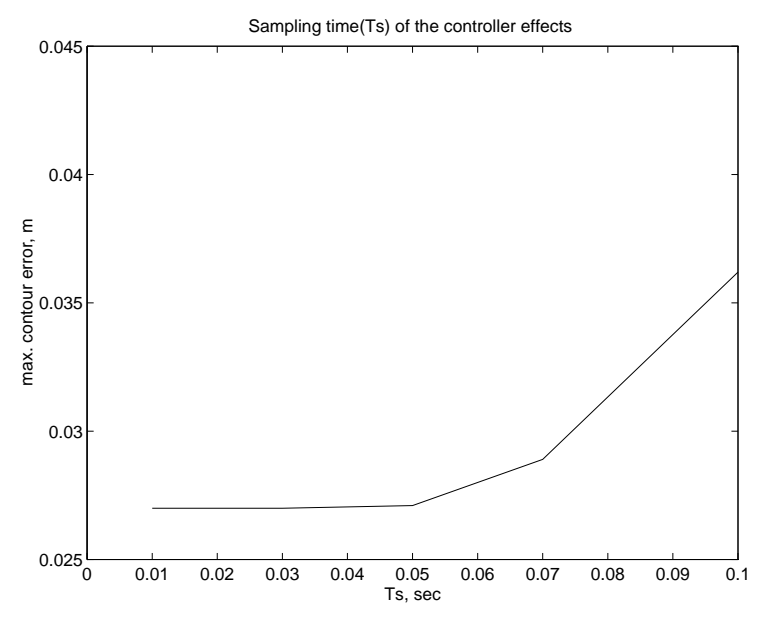

a. Effects of the sample time $T_{s}$ of the servo controller

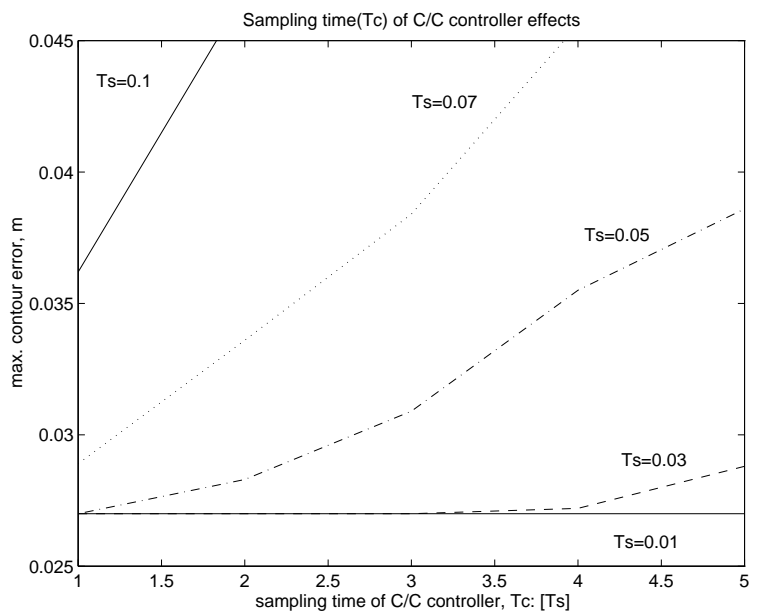

b. Effects of the sample time $T_{c}$ of the cross-coupled controller

Figure 5: The sampling time effects on maximum contour error. For a decentralized cross-coupled controller architecture, with the sample time of the cross-coupled controller equal to the sample time of the servo-level controllers, and no communication delay, we see that the maximum contour error exponentially increases as the sample time $T_{s}$ increases. Part $\mathrm{b}$ of the figure shows how the frequency of execution of the cross-coupled controller affects the contour error. The sample time $T_{s}$ of the servo-level controllers was varied between 0.1 and 0.01 seconds. For each sample time, the frequency of execution of the cross-coupled controller was varied from once per sample to once per five samples. As expected, the effect of this reduced sampling rate is most prominent at low sampling frequencies. The errors presented here are the maximum contour errors for a circular trajectory.

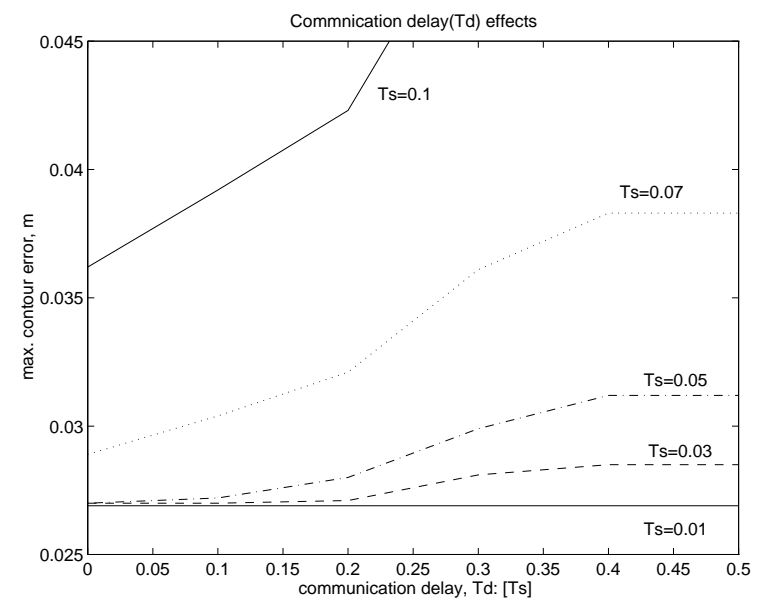

Figure 6: Effects of communication delays on the maximum contour error. For servo-level sampling times between $T_{s}=0.1$ and 0.01 seconds, we varied the communication delay between axes up to one-half of the sampling period with $T_{c}=T_{s}$. As expected, the effect of this communication delay is most prominent at low sampling frequencies. The errors presented here are the maximum contour errors for a circular trajectory. 


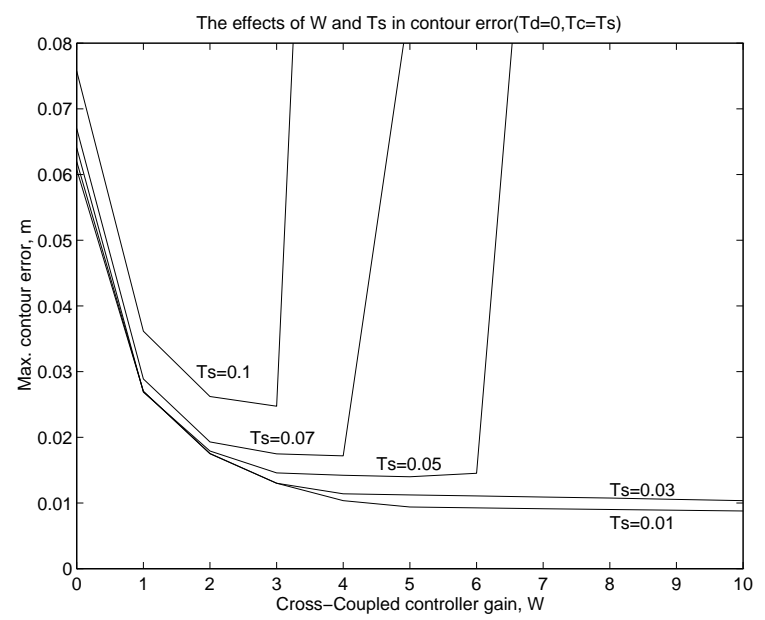

a. $T_{d}=0$

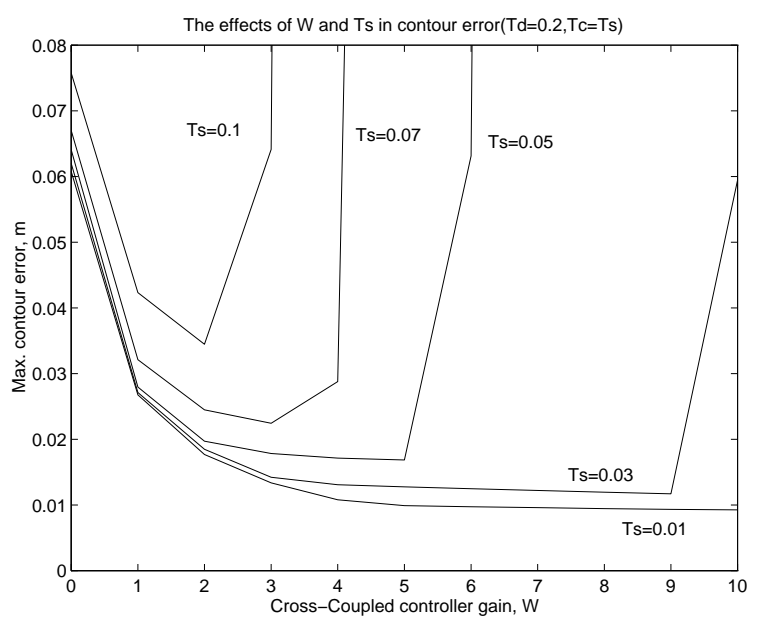

b. $T_{d}=0.2 T_{s}$

Figure 7: Effects of cross-coupling gain $W$ on the contour error. As $W$ increases, the contour error decreases; however, if $W$ is too high, the system becomes unstable. The stability limit is much lower when there is a communication delay between the two axes. The errors presented here are the maximum contour errors for a circular trajectory.

\section{Computing infrastructure architecture}

The computing infrastructure for the running control algorithms can significantly impact performance as well as ease of implementation. For our purposes, we distinguish between uniprocessor, multiprocessor, and distributed system computing environments. A uniprocessor environment has a single memory unit accessed by a single processor, and there is a single thread of computation active at any point in time. A multiprocessor, for our purposes, has several threads of computation active, on several processors, but accessing some common memory repository. Uniprocessor and multiprocessor configurations do not have significant time overheads in terms of communication of information among their components.

In contrast, distributed systems have several threads of computation executing on separate processors - each of which has its local memory. That is, distributed systems resemble a collection of uniprocessors and/or multiprocessors networked using some communication infrastructure. Such environments have significant overheads with regard to communication (which may be needed for synchronization and coupling of algorithms). For flexibility, in our domain of interest, we expect to develop techniques to deploy in distributed environments.

The performance and flexibility of monolithic control algorithms can be enhanced significantly by modularly separating them into several smaller algorithms. In order to achieve the functionality of the original algorithm these "sub-algorithms" may need to share certain variables that effect coordination. In a uniprocessor or a shared memory multiprocessor environment, the sub-algorithms simply use data stored in memory space shared by all the requisite processes (e.g., see [6]). Regardless of the number of processes accessing the shared variables, they are not replicated.

\subsection{Distributed systems}

In situations involving distributed systems (such as decoupled, hierarchical, or decentralized architectures), the sub-algorithms may be executed at different sites. Similar to the monolithic control algorithm each subalgorithm $C_{i}$ is associated with input and output variables represented by vectors $V_{i n}^{i}$ and $V_{\text {out }}^{i}$ respectively. 
Unlike a monolithic algorithm where all inputs are local, here the input variables can be either constants, local variables (i.e., updated by local sensor reading or control task), or remote variables $v_{i j}, j \neq i$ (i.e., updated by remote sensor readings, or outputs of remote control tasks $C_{j}$ ). In order to facilitate access to the variables necessary for the local executions, each site would have a local copy of all input variables. This results in replication of the shared variables, thereby requiring certain consistency constraints enforced among the replicated data. Similarly, each site would also have a copy of all the output variables of the sub-algorithms executing at that site. Thus, for the correct execution of control algorithm $C_{i}$, the value of every input variable $v_{i j}$ must be equal to the value of the corresponding $v_{o u t}^{j}$, one of the outputs of the control algorithm, $C_{j}$. For the control tasks to run correctly, the variables must accurately reflect the environment, and therefore, certain application-dependent time constraints must be met.

The consistency issue described above may be achievable with some associated overhead. In many real time process control situations, especially where time constraints have to be met, these overheads have to be considered. Fortunately, in actual manufacturing environment, consistency at all times is not required. It may often suffice if an input variable $v_{i j}$ represents the latest value of the corresponding $v_{\text {out }}^{j}$ only at the time when it is used by the control task. This gives us some leeway to seek a feasible distributed real time data management strategy with replication across sites.

\section{$5.2 \quad$ Effecting distributed consistency}

At every site we maintain a table which contains an entry for shared variables associated with all the subalgorithms executing at that site. For each variable we store its unique id, value, version number, and a "site list." The site list indicates every remote site which uses the output variable. Input variables have an empty site list. The task itself is unaware of the source of the input variables (local or remote) thus allowing for autonomy.

For every output variable $v_{\text {out }}^{i}$ written out to the table its version number is incremented and its value is multicast to all sites in the site list [9]. On receiving the multicast, the value of the corresponding variable at each site is updated. This communication introduces a time delay $T_{d}$ during which the input variable is inconsistent with regard to the corresponding output variable. The maximum delay, $T_{d}$, that can be tolerated is determined by the different types of coordination constraints necessitated by the application environment. This imposes a time constraint within which the message must be sent.

For the purpose of communication, an output queue may be maintained for messages that need to be sent to remote sites in order to propagate the output variables. The priorities assigned to the elements in the queue could be based on the earliest deadlines associated with all destinations of a message. The deadlines are based on some global concept of time and we assume that the sites have synchronized clocks. It is also assumed that the network supports multicasting (and if not, it would need to be implemented). If the network supports priority message handling, the earliest deadline of the message could be used to determine the priority. By this approach, the most urgent messages are sent earlier - in keeping with the Earliest Deadline First (EDF) scheduling approach. Certainly, other forms of real-time scheduling can be considered as well.

Among the required processes, one would read the most recent values of the input variables from the shared table and provide them to the control algorithms. Another process would update the version numbers and multicast messages for every output variable. To ensure correctness, these processes should be executed every $T_{s}$ seconds where $T_{s}$ is the period of the control algorithm with which they are associated. All the processes executed at a site must be scheduled so that they meet the real time requirements. Also it should be noted that the software and hardware requirements place limitations on the extent and the feasibility of modularization. More information on the necessary computing infrastructure for a reconfigurable machining system can be found in [21, 24]. 


\section{Conclusions and future work}

We are interested in designing control systems for modular, reconfigurable machine tools, and desire that the control systems should also be modular and reconfigurable. Future machine tool modules may come pre-equipped with the necessary control hardware and software for that particular module, along with a network connection so that the assembled control modules in a machine can communicate and coordinate their actions. In order to achieve this vision, reconfigurable and decentralized control strategies will be required.

In this paper, four different architectures for distributed control of machine tools - centralized, decoupled, hierarchical, and decentralized - have been defined and examined. The advantages and disadvantages of each architecture, from a performance and reconfigurability point of view, have been discussed. The different types of time delays present in each type of system have been considered, and their effects analyzed both mathematically and through simulations. A simple two-axis contouring system was considered as an example throughout the paper, and the effect of different types of time delays on the performance of the system was shown.

From these analytic and simulation results on the effect of timing delays on the system performance, the constraints on the distributed computation and communication systems can be derived. We proposed an approach to manage coordination in the systems using a distributed real-time data repository. Some important consistency and temporal constraints on the data were defined. Thereafter, we suggested an approach to satisfy the real-time constraints imposed by the control performance criteria in the distributed system.

This paper represents our preliminary definition and identification of the problems that will be encountered in the design and construction of modular, distributed real-time control systems for machining systems. We are working to extend our analytic results to more types of machining systems, including more complex multi-axis systems. We are also working on implementing the protocols described in Section 5 to determine their effectiveness experimentally, and are coordinating with other control and software projects within the Center for Reconfigurable Machining Systems to integrate our results within both the physical testbed and the control design software.

\section{References}

[1] Y. Altintaş and W. K. Munasinghe. Modular CNC design for intelligent machining, part 2: Modular integration of sensor based milling process monitoring and control tasks. ASME Journal of Manufacturing Science and Engineering, 118:514-521, November 1996.

[2] Y. Altintaş, N. Newell, and M. Ito. Modular CNC design for intelligent machining, part 1: Design of a hierarchical motion control module for CNC machine tools. ASME Journal of Manufacturing Science and Engineering, 118:506-513, November 1996.

[3] J.-D. Decotignie, D. Auslander, and M. Moreaux. Fieldbus based integrated communication and control systems. In Proceedings of the International Workshop on Advanced Motion Control, pages 541-546, Tsu, Japan, 1996.

[4] L. Ferrarini, G. Ferretti, C. Maffezzoni, and Gian Antonio Magnani. Hybrid modeling and simulation for the design of an advanced industrial robot controller. IEEE Robotics and Automation Magazine, 4(2):45-51, June 1997.

[5] G. F. Franklin, J. D. Powell, and M. L. Workman. Digital Control of Dynamic Systems. Addison-Wesley, second edition, 1992. 
[6] M. Gretz and P. Khosla. Onika: A multilevel human-machine interface for real-time sensor-based systems. In ASCE/SPACE: 4th Int'l Conference and Expo on Engineering Construction and Operations in Space, 1994.

[7] S. Jee and Y. Koren. Friction compensation in feed drive systems using an adaptive fuzzy logic control. In Proceedings of the International Mechanical Engineering Congress and Exposition (Dynamic Systems and Control Division), pages 885-893, Chicago, 1994.

[8] S. C. Jee and Yoram Koren. Self-organizing fuzzy logic control for friction compensation in feed drives. In Proceedings of the American Control Conference, pages 205-209, 1995.

[9] P. Jensen, N. Soparkar, and M. Tayara. Towards distributed real-time concurrency and coordination control. In S. Jajodia and L. Kerschberg, editors, Advanced Transaction Models and Architectures. Kluwer Academic, 1997.

[10] Y. Koren. Cross-coupled biaxial computer control for manufacturing systems. ASME Journal of Dynamic Systems, Measurement, and Control, 102:265-272, 1980.

[11] Y. Koren. Computer Control of Manufacturing Systems. Mc-Graw Hill, 1983.

[12] Y. Koren and C. C. Lo. Advanced controllers for feed drives. Annals of CIRP, 41(2):689-698, 1992.

[13] Y. Koren, Z. J. Pasek, A. G. Ulsoy, and U. Benechetrit. Real-time open control architectures and system performance. Annals of the CIRP, 45(1):377-380, 1996.

[14] Y. Koren, Z. J. Pasek, A. G. Ulsoy, and P. K. Wright. Timing and performance of open architecture controllers. In Proceedings of the International Mechanical Engineering Congress and Exposition (Dynamic Systems and Control Division), pages 283-290, 1996.

[15] Y. Koren and A. G. Ulsoy. Engineering research center for reconfigurable machining systems. http://erc.engin.umich.edu.

[16] R. G. Landers. Supervisory Machining Control: A Design Approach plus Chatter Analysis and Force Control Components. PhD thesis, University of Michigan, 1997.

[17] R. G. Landers and A. G. Ulsoy. Machining force control including static, non-linear effects. In Proceedings of the Japan/USA Symposium on Flexible Automation, pages 983-990, 1996.

[18] M. Malek-Zavarei and M. Jamshidi. Time-Delay Systems Analysis, Optimization, and Application. North-Holland, 1987.

[19] Manufacturing Automation Laboratories Inc. http://www.malinc.com.

[20] D. Song, T. Divoux, and F. Lepage. Design of the distributed architecture of a machine-tool using fip fieldbus. In Proceedings of the IEEE Conference on Application-Specific Systems, Architectures, and Processors, pages 250-260, 1996.

[21] N. Soparkar and D. Tilbury. Decentralized data and real-time control. Journal of Systems and Software, 1997. Submitted to the Special Issue on Real-Time Active Databases: Theory and Practice.

[22] K. Srinivasan and P. K. Kulkarni. Cross-coupled control of biaxial feed drive mechanisms. ASME Journal of Dynamic Systems, Measurement, and Control, 112:225-232, 1990.

[23] J. A. Stankovic. Misconceptions about real-time computing. IEEE Computer, October 1988. 
[24] M. Tayara, N. Soparkar, J. Yook, and D. Tilbury. Real-time data and coordination control for reconfigurable manufacturing systems. In A. Bestavros and V. Wolfe, editors, Real-Time Database and Information Systems: Research Advances. Kluwer Academic, Norwell, MA, 1997.

[25] D. Tilbury and N. Soparkar. Benchmarking report for ERC project 2.2: Distributed real-time control for reconfigurable manufacturing systems. Available through the ERC office, August 1997.

[26] A. Galip Ulsoy and Yoram Koren. Control of machining processes. ASME Journal of Dynamic Systems, Measurement, and Control, 115:301-308, 1993.

[27] B. Wittenmark, J. Nilsson, and M. Törngren. Timing problems in real-time control systems. In Proceedings of the American Control Conference, pages 2000-2004, Seattle, WA, June 1995.

[28] H. Zhang, J. Ni, and H. Shi. Machining chatter suppression by means of spindle speed variation. In Proceedings of the first S.M. Wu Symposium on Manufacturing Science, volume 1, pages 161-167. Society of Manufacturing Engineers, 1994. 